\title{
Sequential Auctions for Objects with Common and Private Values
}

\author{
Shaheen Fatima \\ Department of Computer \\ Science \\ University of Liverpool \\ Liverpool L69 7ZF, U.K. \\ shaheen@csc.liv.ac.uk
}

\author{
Michael Wooldridge \\ Department of Computer \\ Science \\ University of Liverpool \\ Liverpool L69 7ZF, U.K. \\ mjw@csc.liv.ac.uk
}

\author{
Nicholas R. Jennings \\ School of Electronics and \\ Computer Science \\ University of Southampton \\ Southampton SO17 1BJ, U.K. \\ nrj@ecs.soton.ac.uk
}

\begin{abstract}
Sequential auctions are an important mechanism for buying/selling multiple objects. Existing work has studied sequential auctions for objects that are exclusively either common value or private value. However, in many real-world cases an object has both features. Also, in such cases, the common value component (which is the same for all bidders) depends on how much each bidder values the object. Moreover, an individual bidder generally does not know the true common value, since it may not know how much the other bidders value it. On the other hand, a bidder's private value is independent of the others' private values. Given this, we study settings that have both common and private value elements by treating each bidder's information about the common value as uncertain. We first determine equilibrium bidding strategies for each auction in a sequence using English auction rules. On the basis of this equilibrium, we analyse the efficiency of auctions. Specifically, we show that the inefficiency that arises as a result of uncertainty about the common values can be reduced if the auctioneer makes its information about the common value known to all bidders. Moreover, our analysis also shows that the efficiency of auctions in an agent-based setting is higher than that in an all-human setting.
\end{abstract}

\section{Categories and Subject Descriptors}

I.2.11 [Distributed Artificial Intelligence]: Multiagent Systems; K.4.4 [Computers and Society]: Electronic Commerce

\section{Keywords}

Game-theory, Multi-object auctions.

Permission to make digital or hard copies of all or part of this work for personal or classroom use is granted without fee provided that copies are not made or distributed for profit or commercial advantage and that copies bear this notice and the full citation on the first page. To copy otherwise, to republish, to post on servers or to redistribute to lists, requires prior specific permission and/or a fee.

AAMAS'05 July 25-29, 2005, Utrecht, Netherlands

Copyright 2005 ACM 1-59593-094-9/05/0007 ...\$5.00.

\section{INTRODUCTION}

Market-based mechanisms such as auctions are now being widely studied as a means of buying/selling resources in multiagent systems. This uptake is occurring because auctions are both simple and have a number of desirable properties (typically the most important of which are their ability to generate high revenues to the seller and to allocate resources efficiently [17, 3, 19]). Now, in many cases the number of objects to be auctioned is greater than one. In such cases, there are two types of auctions that can be used: combinatorial auctions [16] and sequential auctions $[4,2]$. The former are used when the objects for sale are available at the same time, while the latter (which is the main focus of this paper) are used when the objects become available at different points in time.

In the sequential case, the auctions are conducted at different times, therefore a bidder may participate in more than one of them. In such a scenario, it has been shown that although there is only one object being auctioned at a time, the bidding behaviour for any individual auction strongly depends on the auctions that are yet to be conducted $[4,2]$. For example, consider sequential auctions for oil exploration rights. In this scenario, the price an oil company will pay for a given area is affected not only by the area that is available in the current round, but also by the areas that will become available in subsequent rounds of leasing. Thus, it would be foolish for a bidder to spend all the money set aside for exploration on the first round of leasing, if potentially even more favourable sites are likely to be auctioned off subsequently.

Against this background, a key problem in the area is to study the strategic behaviour of bidders in each individual auction. To date, considerable research effort has been devoted to this problem, but a key drawback is that it deals with objects that are either exclusively common value (an object is worth the same to all bidders) [1] or exclusively private value (different bidders value the same object differently) $[20,6,15,4]$. Also, several of them assume complete information about these values is available to the bidders $[15,1]$. However, most real-world auctions are neither exclusively private nor common value, but involve an element of both [8]. Again, consider the above example of auctioning oil drilling rights. This is, in general, treated as a common value auction. But private value differences may arise, for example, when a superior technology enables some firm to exploit the rights better than others. Also, in such cases, the common value depends on how much each bidder values the object. Moreover, an individual bidder generally does not know the true common value, since it may not know how much the other bidders value it.

Given this, our objective is to study sequential auctions that have both common and private value elements by treating each bidder's 
information about the common value as uncertain. We study the case where each bidder needs at most one object. Each object is modelled with a two-dimensional signal: one for its common value and the other for its private value. The auctions are conducted using English auction rules. We first determine equilibrium bidding strategies for each individual auction in a sequence. On the basis of this equilibrium, we study auction efficiency. We focus on efficiency because in the context of objects with common and private values, it is of major importance (because although it is possible to have efficient auctions for objects that are either only common value or only private value, it is not possible to have efficient auctions for objects that have both elements $[9,3]$ - see Section 7 for more details). We then show how uncertainty and competition (i.e., the number of bidders) affect auction efficiency. Here the role of competition is important in an agent-based auction setting than in a human setting because the former case leads to more competition [14].

The two key results of our analysis are as follows. First, we show that for each auction in a sequence, efficiency decreases with uncertainty (we also provide a means of reducing inefficiency). Second, we show that an increase in competition increases auction efficiency. Since competition in an agent-based setting is higher than that in an all-human setting, this result implies that efficiency of auctions in an agent-based setting is higher than in a human setting.

The remainder of the paper is organized as follows. Section 2 describes the auction setting. Section 3 determines equilibrium bidding strategies. Section 4 studies the effect of uncertainty on the equilibrium outcome. Section 5 studies the effect of disclosure of the auctioneer's information and Section 6 that of competition on the auction efficiency. Section 7 provides a discussion of related literature and Section 8 concludes.

\section{THE SEQUENTIAL AUCTIONS MODEL}

Single object auctions that have both private and common value elements have been studied in [5]. We therefore adopt this basic model and extend it to cover the multiple objects case. Before doing so, however, we give an overview of the basic model.

\subsection{Single object}

A single object auction is modelled in [5] as follows. There are $n \geq 3$ risk neutral bidders. The common value $\left(V_{1}\right)$ of the object to the $n$ bidders is equal, but initially the bidders do not know this value. However, each bidder receives a signal that gives an estimate of this common value. Bidder $i=1, \ldots, n$ draws an estimate $\left(v_{i 1}\right)$ of the object's true value $\left(V_{1}\right)$ from the probability distribution function $Q(v)$ with support $\left[v_{L}, v_{H}\right]$. Although different bidders may have different estimates, the true value $\left(V_{1}\right)$ is the same for all bidders and is modelled as the average of the bidders' signals:

$$
V_{1}=\frac{1}{n} \sum_{i=1}^{n} v_{i 1}
$$

Furthermore, each bidder has a cost which is different for different bidders and this cost is its private value. For $i=1, \ldots, n$, let $c_{i 1}$ denote bidder $i$ 's signal for this private value which is drawn from the distribution function $G(c)$ with support $\left[c_{L}, c_{H}\right]$ where $c_{L} \geq 0$ and $v_{L} \geq c_{H}$. Cost and value signals are independently and identically distributed across bidders. Henceforth, we will use the term value to refer to common value and cost to refer to private value.

If bidder $i$ wins the object and pays $b$, it gets a utility of $V_{1}-$ $c_{i 1}-b$, where $V_{1}-c_{i 1}$ is $i$ 's surplus. Each bidder bids so as to maximize its utility. Note that bidder $i$ receives two signals $\left(v_{i}\right.$ and $c_{i}$ ) but its bid has to be a single number. Hence, in order to determine their bids, bidders need to combine the two signals into a summary statistic. This is done as follows. For $i$, a one-dimensional summary signal, called $i$ 's surplus ${ }^{1}$, is defined as:

$$
S_{i 1}=v_{i 1} / n-c_{i 1}
$$

which allows $i$ 's optimal bids to be determined in terms of $S_{i 1}$ (see [5] for more details about the problems with two signals and why a one-dimensional surplus is required). In order to rank bidders from low to high valuations, $Q(v)$ and $G(c)$ are assumed to be $\log$ concave $^{2}$. Under this assumption, the conditional expectations $E(v \mid S=x)$ and $E(v \mid S \leq x)$ are non-decreasing in $x$. Furthermore, $E(c \mid S=x)$ and $E(c \mid S \leq x)$ are non-increasing in $x$. In other words, the bidders can be ranked from low to high values on the basis of their surplus. We extend this model to $m>1$ objects.

\subsection{Multiple objects}

For each of the $m>1$ objects, the bidders' values are independently and identically distributed and so are their costs. There are $m$ distribution functions for the common values, one for each object. Likewise, there are $m$ distribution functions for the costs, one for each object. For $j=1, \ldots, m$, let $Q_{j}: R_{+} \rightarrow[0,1]$ denote the distribution function for the value of the $j$ th object and $G_{j}: R_{+} \rightarrow[0,1]$ that for its cost. Thus, each bidder receives its value signal for the $j$ th object from $Q_{j}$ and its cost signal from $G_{j}$.

Furthermore, each bidder receives the cost and value signals for an auction just before that auction begins. The signals for the $j$ th object are received only after the $(j-1)$ previous auctions have been conducted. Consequently, although the bidders know the distribution functions from which the signals are drawn, they do not know the actual signals for the $j$ th object until the previous $(j-1)$ auctions are over.

The $m$ objects are sold one after another in $m$ auctions that are conducted using English auction rules. Furthermore, each bidder can win at most one object. The winner for the $j$ th object does not participate in the remaining $m-j$ auctions. Thus, if $n$ agents participate in the first auction, the number of agents for the $j$ th auction is $(n-j+1)$.

For objects $j=1, \ldots, m$ and bidders $i=1, \ldots, n$, let $v_{i j}$ and $c_{i j}$ denote the common and private values respectively. The true common value of the $j$ th object (denoted $V_{j}$ ) is:

$$
V_{j}=\frac{1}{n-j+1} \sum_{i=1}^{n-j+1} v_{i j}
$$

For objects $j=1, \ldots, m$ and bidders $i=1, \ldots, n$, let $S_{i j}$ denote $i$ 's surplus for object $j$ where

$$
S_{i j}=v_{i j} /(n-j+1)-c_{i j}
$$

Note that the values/costs for our model are not correlated. Such correlations occur across objects, if for a bidder (say $i$ ) the value/cost of object $j=2, \ldots, m$ can be determined on the basis of $i$ 's value/cost signal for the first object. However, in many cases such

\footnotetext{
${ }^{1}$ Note that $i$ 's true surplus is $V_{1}-c_{i 1}$ which is equal to $v_{i 1} / n-$ $c_{i 1}+\sum_{j \neq i} v_{j 1} / n$. But since $v_{i 1} / n-c_{i 1}$ depends on $i$ 's signals while $\sum_{j \neq i} v_{j 1} / n$ depends on the other bidders' signals, the term ' $i$ 's surplus' is also used to mean $v_{i 1} / n-c_{i 1}$.

${ }^{2} \log$ concavity means that the natural $\log$ of the densities is concave. This restriction is met by many commonly used densities like uniform, normal, chi-square, and exponential, and it ensures that optimal bids are increasing in surplus. Again see [5] for more details.
} 
a direct relation between the objects may not exist. Hence, we focus on the case where different objects have different distribution functions. Furthermore, although each bidder knows the distribution functions from which the values/costs are drawn before the first auction begins, it receives its signals for an object only just before the auction for that object begins.

\section{EQUILIBRIUM BIDDING STRATEGIES}

The $m$ objects are auctioned in $m$ separate English auctions that are conducted sequentially. The English auction rules are as follows. The auctioneer continuously raises the price, and bidders publicly reveal when they withdraw from the auction. Bidders who drop out from an auction are not allowed to re-enter that auction. A bidder's strategy for the $j$ th (for $j=1, \ldots, m-1$ ) auction depends on how much profit it expects to get from the $(m-j)$ auctions yet to be conducted. However, since there are only $m$ objects there are no more auctions after the $m$ th one. Thus, a bidder's strategic behaviour during the last auction is the same as that for a single object English auction. Equilibrium bidding strategies for a single object of the type described in Section 2 have been obtained in [5]. We therefore briefly summarize these strategies and then determine equilibrium for our $m$ objects case.

\subsection{Single object}

For a single object with value $V_{1}$, the equilibrium obtained in [5] is as follows. A bidder's strategy is described in terms of its surplus and indicates how high the bidder should go before dropping out. Since $n \geq 3$, the prices at which some bidders drop out convey information (about the common value) to those who remain active. Suppose $k$ bidders have dropped out at bid levels $b_{1} \leq \ldots \leq b_{k}$. A bidder's (say $i$ 's) strategy is described by functions $B_{k}\left(S_{i} ; b_{1} \ldots b_{k}\right)$, which specify how high it must bid given that $k$ bidders have dropped out at levels $b_{1} \ldots b_{k}$ and given that its surplus is $S_{i}$. The $n$-tuple of strategies $(B(\cdot), \ldots, B(\cdot))$ with $B(\cdot)$ defined in Equation 4, constitutes a symmetric equilibrium of the English auction.

$$
\begin{aligned}
B_{0}\left(x_{i}\right)= & E\left(v_{i}-c_{i} \mid S_{i}=x_{i}\right) \\
B_{k}\left(x_{i} ; b_{1} \ldots b_{k}\right)= & \frac{n-k}{n} E\left(v_{i} \mid S_{i}=x_{i}\right) \\
+\frac{1}{n} \sum_{j=0}^{k-1} E\left(v_{i} \mid\right. & \left.B_{j}\left(S_{i} ; b_{1}, \ldots, b_{j}\right)=b_{j+1}\right) \\
& -E\left(c_{i} \mid S_{i}=x_{i}\right)
\end{aligned}
$$

where $x_{i}$ is $i$ 's surplus. The intuition for Equation 4 is as follows. Given its surplus and the information conveyed in others' drop out levels, the highest a bidder is willing to go is given by the expected value of the object, assuming that all other active bidders have the same surplus. For instance, consider the bid function $B_{0}\left(S_{i}\right)$ which pertains to the case when no bidder has dropped out yet. If all other bidders were to drop out at level $B_{0}\left(S_{0}\right)$, then $i$ 's expected payoff $\left(e p=V_{1}-c_{i}-B_{0}\left(S_{0}\right)\right.$ ) would be:

$$
\begin{aligned}
e p & =S_{i}+\frac{n-1}{n} E\left(v \mid S=S_{0}\right)-B_{0}\left(S_{0}\right) \\
& =S_{i}+\frac{n-1}{n} E\left(v \mid S=S_{0}\right)-E\left(v-c \mid S=S_{0}\right) \\
& =S_{i}-S_{0}
\end{aligned}
$$

Using strategy $B_{0}, i$ remains active until it is indifferent between winning and quitting. Similar interpretations are given to $B_{k}$ for $k \geq 1$; the only difference is that these functions take into account the information conveyed in others' drop out levels.
Let $f_{1}^{n}$ denote the first order statistic of the surplus for the $n$ bidders and let $s_{1}^{n}$ denote the second order statistic. $f_{1}^{n}$ and $s_{1}^{n}$ are obtained from the distribution functions $Q_{1}$ and $G_{1}$. For the above equilibrium, the bidder with the highest surplus wins. The expected selling price, the winner's expected profit, the total expected surplus, and the expected revenue are as follows [5]. The expected selling price (denoted $E\left(P_{w}\right)$ ) is:

$$
E\left(P_{w}\right)=E\left(s_{1}^{n}\right)
$$

The winner's expected profit (denoted $E\left(\pi_{w}\right)$ ) is:

$$
E\left(\pi_{w}\right)=E\left(f_{1}^{n}\right)-E\left(s_{1}^{n}\right)
$$

The total expected surplus (denoted $E(W)$ ) is the surplus that gets split between the auctioneer and the winning bidder. It is:

$$
E(W)=E\left(V_{1}\right)-E\left(c_{w}\right)
$$

where $E\left(c_{w}\right)$ denotes the expected cost of the winner. Finally, the expected revenue (denoted $E(R)$ ) is the difference between the surplus $(E(W))$ and the winner's expected profit $\left(E\left(\pi_{w}\right)\right)$. This revenue is:

$$
E(R)=E(W)-E\left(\pi_{w}\right)
$$

On the basis of the above equilibrium for a single object, we now determine equilibrium for sequential auctions for the $m$ objects defined in Section 2.

\subsection{Multiple objects}

We will denote the first order statistic of the surplus for the $j$ th (for $j=1, \ldots, m)$ auction as $f_{j}^{n-j+1}$ and the second order statistic as $s_{j}^{n-j+1}$. The following theorem characterises the equilibrium for $m>1$ objects.

THEOREM 1. For $j=1, \ldots, m$, let $\beta_{j}=1 /(n-j+1)$ and let $\alpha_{j}$ be defined as:

$$
\alpha_{j}=\sum_{y=j}^{m}\left(\beta_{y} \times X_{y}\right)
$$

where $X_{y}=E\left(f_{y}^{n-y+1}\right)-E\left(s_{y}^{n-y+1}\right)+X_{y+1}$ for $y=1, \ldots, m$ and $X_{m+1}=0$. Then the $n$-tuple of strategies $(B(\cdot), \ldots, B(\cdot))$ with $B(\cdot)$ defined in Equation 9 constitutes an equilibrium for the $j$ th (for $j=1, \ldots,(m-1)$ ) auction at a stage where $k$ bidders have dropped out:

$$
\begin{array}{rll}
B_{0}^{j}\left(x_{i j}\right)= & E\left(v_{i j}-c_{i j} \mid S_{i j}=x_{i j}\right)-\alpha_{j+1} \\
B_{k}^{j}\left(x_{i j} ; b_{1}, \ldots, b_{k}\right)= & \frac{n-j+1-k}{n-j+1} E\left(v_{i j} \mid S_{i j}=x_{i j}\right) \\
& & -E\left(c_{i j} \mid S_{i j}=x_{i j}\right) \\
+\frac{1}{n-j+1} \quad \sum_{y=0}^{k-1} & E\left(v_{i j} \mid B_{y}\left(S_{i j} ; b_{1}, \ldots, b_{y}\right)=b_{y+1}\right) \\
& & -\alpha_{j+1}
\end{array}
$$

For the last auction, the equilibrium is as given in Equation 4 with $n$ replaced with $(n-m+1)$.

PROOF. We consider each of the $m$ auctions by reasoning backwards.

- mth auction. To begin, consider the mth auction for which there are $(n-m+1)$ bidders. Since this is the last auction, an agent's bidding behaviour is the same as that for the single object case. Hence, the equilibrium for this auction is the same as that in Equation 4 with $n$ replaced with 
$(n-m+1)$. For $j=1, \ldots, m$, let $\alpha_{i j}$ denote an agent's cumulative ex-ante expected profit from auctions $j$ to $\mathrm{m}$. Recall that although the bidders know the distribution (from which the cost and value signals are drawn) before the first auction begins, they draw the signals for the jth auction only after the $(j-1)$ earlier auctions end. Since $\alpha_{i j}$ is the ex-ante expected profit (i.e., it is computed before the bidders draw their signals for the jth auction), it is the same for all participating bidders. Thus, we will simplify notation by dropping the subscript $i$ and denote $\alpha_{i j}$ simply as $\alpha_{j}$ We know from Equation 6 that:

$$
\alpha_{m}=\frac{1}{n-m+1}\left(E\left(f_{m}^{n-m+1}\right)-E\left(s_{m}^{n-m+1}\right)\right.
$$

This is because all the $(n-m+1)$ agents that participate in the mth auction have ex-ante identical chances of winning it. Note that the right hand side of Equation 10 does not depend on $i$. In other words, since bidders receive their signals for the mth auction after the $(m-1)$ th auction, the ex-ante expected profit for the mth auction (before the $(m-1)$ th auction ends) is the same for all the $(n-m+1)$ bidders.

- $(m-1)$ th auction. Consider the $(m-1)$ th auction. During this auction, a bidder bids $b$ if $\left(V_{m-1}-c_{m-1}-b \geq \alpha_{m}\right)$ or

$$
b \leq V_{m-1}-c_{m-1}-\alpha_{m}
$$

Hence, a symmetric equilibrium for the $(m-1)$ th auction is obtained by substituting $j=m-1$ in Equation 9. We know from Equation 5, that the expected selling price for the single object case is the second order statistic of the surplus. The difference between the equilibrium bids for the single object case and the $(m-1)$ th auction of the $m$ objects case is $\alpha_{m}$ (see Equations 4 and 9). Hence, the expected selling price for the $(m-1)$ th auction is $E\left(s_{m-1}^{n-m+2}\right)-\alpha_{m}$. This implies that the winner's expected profit for the $(m-1)$ th auction is:

$$
\begin{aligned}
E\left(\pi_{w(m-1)}\right)= & E\left(f_{m-1}^{n-m+2}\right)-E\left(s_{m-1}^{n-m+2}\right)+ \\
& \alpha_{m}
\end{aligned}
$$

- First $(m-2)$ auctions. Consider the jth auction where $1 \leq$ $j \leq m-2$. We now find $\alpha_{1}, \ldots, \alpha_{m-1}$. Since the number of bidders for the jth auction is $(n-j+1)$ and each bidder has ex-ante equal chances of winning, the probability that a bidder wins the $j$ th (for $j=1, \ldots, m$ ) auction (denoted $\beta_{j}$ ) is:

$$
\beta_{j}=1 /(n-j+1)
$$

This implies that, for $j=1, \ldots, m, \alpha_{j}$ is:

$$
\alpha_{j}=\beta_{j} E\left(\pi_{w j}\right)+\alpha_{j+1}
$$

Generalising Equation 12 to the first $(m-1)$ auctions, we get the winner's expected profit $\left(E\left(\pi_{w j}\right)\right)$ as:

$$
E\left(\pi_{w j}\right)=E\left(f_{j}^{n-j+1}\right)-E\left(s_{j}^{n-j+1}\right)+\alpha_{j+1}
$$

Consequently, a bidder's optimal bid for the jth auction is obtained by discounting the single object equilibrium bid by $\alpha_{j+1}$. Hence, we get the equilibrium bids in Equation 9.

THEOREM 2. For the $j$ th (for $j=1, \ldots, m$ ) auction, the expected selling price (denoted $E\left(P_{w j}\right)$ ) is:

$$
\forall_{j=1}^{m-1} E\left(P_{w j}\right)=E\left(s_{j}^{n-j+1}\right)-\alpha_{j+1}
$$

$$
E\left(P_{w m}\right)=E\left(s_{m}^{n-m+1}\right)
$$

The winner's expected profit $\left(E\left(\pi_{w j}\right)\right)$, the total expected surplus $\left(E\left(W_{j}\right)\right)$, and the expected revenue $\left(E\left(R_{j}\right)\right)$ are:

$$
\begin{aligned}
\forall_{j=1}^{m-1} E\left(\pi_{w j}\right)= & E\left(f_{j}^{n-j+1}\right)-E\left(s_{j}^{n-j+1}\right) \\
& +\alpha_{j+1} \\
E\left(\pi_{w m}\right)= & E\left(f_{m}^{n-m+1}\right)-E\left(s_{m}^{n-m+1}\right) \\
\forall_{j=1}^{m} E\left(W_{j}\right)= & E\left(V_{j}\right)-E\left(c_{w j}\right) \\
\forall_{j=1}^{m-1} E\left(R_{j}\right)= & E\left(W_{j}\right)-\left(E\left(f_{j}^{n-j+1}\right)-E\left(s_{j}^{n-j+1}\right)\right. \\
& \left.+\alpha_{j+1}\right) \\
E\left(R_{m}\right)= & E\left(W_{m}\right)-\left(E\left(f_{m}^{n-m+1}\right)-E\left(s_{m}^{n-m+j}\right)\right.
\end{aligned}
$$

where $E\left(c_{w j}\right)$ denotes the expected cost of the winner for the jth auction.

PROOF. It is important to note that for the $j$ th $(j=1, \ldots, m)$ the bids in Theorem 1 are similar to those in Equation 4 (for the single object case), except that each bid in the former case is obtained from the corresponding bid in the latter by shifting the latter by the constant $\alpha_{j+1}$. Since $\alpha_{j+1}$ is the same for all participating bidders, the relative positions of bidders for each of the $m$ auctions remains the same as that for the corresponding single object case. Hence, the winner for the $j$ th (for $j=1, \ldots, m$ ) auction is the bidder with the highest surplus for that auction. Consequently, the expected selling price for the $j$ th (for $j=1, \ldots, m-1$ ) auction is:

$$
E\left(P_{w j}\right)=E\left(s_{j}^{n-j+1}\right)-\alpha_{j+1}
$$

For the last auction (which is similar to a single object auction), the selling price is:

$$
E\left(P_{w m}\right)=E\left(s_{m}^{n-m+1}\right)
$$

The mth auction is identical to the single object case. Hence, the expected profit for this auction is: $E\left(f_{m}^{n-m+1}\right)-E\left(s_{m}^{n-m+1}\right)$. The relative positions of the bidders for the $j$ th (for $j=1, \ldots, m-1$ ) auction of the $m$ object case and the corresponding single object auction are the same. Since the difference between the selling price of each of the first $m-1$ objects and the corresponding single object case is $\alpha_{j+1}$, the winner's expected profit for the jth auction is:

$$
E\left(f_{j}^{n-j+1}\right)-E\left(s_{j}^{n-j+1}\right)+\alpha_{j+1}
$$

For $j=1, \ldots, m$ the total expected surplus $\left(E\left(W_{j}\right)\right)$ that gets split between the auctioneer and the winning bidder is obtained from Equation 7 as:

$$
E\left(W_{j}\right)=E\left(V_{j}\right)-E\left(c_{w j}\right)
$$

For $j=1, \ldots, m$, the expected revenue $\left(E\left(R_{j}\right)\right)$ for the $j$ th auction is the difference between the surplus $\left(E\left(W_{j}\right)\right)$ and the winner's expected profit $\left(E\left(\pi_{w j}\right)\right)$. Thus, we have:

$$
\begin{aligned}
\forall_{j=1}^{m-1} E\left(R_{j}\right)= & E\left(W_{j}\right)-\left(E\left(f_{j}^{n-j+1}\right)-E\left(s_{j}^{n-j+1}\right)\right. \\
& \left.+\alpha_{j+1}\right) \\
E\left(R_{m}\right)= & E\left(W_{m}\right)-\left(E\left(f_{m}^{n-m+1}\right)-E\left(s_{m}^{n-m+1}\right)\right)
\end{aligned}
$$

Recall from Section 2, that the common value of an object depends on the value signals of all the bidders. Also, although all the bidders know the probability distributions from which the signals are 
drawn, each bidder knows only the signals that it draws and not those of the others'. This lack of information results in an uncertainty about the common values of the objects. In the following section, we study the effect of this uncertainty on the allocative efficiency. We also show how competition affects efficiency.

\section{THE EFFECT OF UNCERTAINTY}

Consider the allocative efficiency of the equilibrium obtained in Section 3. The efficiency of an auction is, in general, measured in terms of the total surplus (that gets split between the auctioneer and the winning bidder) it generates [7]. The larger the surplus, the higher are the gains that are split between the auctioneer and the winning bidder, and the more efficient the auction. We know from Equation 15, that the total expected surplus for the $j$ th auction is decreasing in the cost of the winner for that auction. Also, from the equilibrium given in Theorem 1, we know that the winner for the $j$ th auction (for $j=1, \ldots, m$ ) is the bidder that has the highest surplus for that auction. Although the winner has the highest surplus, it may not necessarily have the lowest cost. This gives rise to inefficiency in the allocation of the objects. In this section, we determine the relation between the efficiency of an auction and the uncertainty (described in detail below) about the common value of the object sold in that auction.

Recall that a bidder's cost is its private value component and does not depend on the other bidders' signals. But the common value component depends on the signals of all the bidders (see Equation 2). Although a bidder only knows its value (from its value signal) it is uncertain about the values of the others. In order to study the effect of uncertainty on the equilibrium outcomes of the $m$ auctions, a weight $(\gamma \geq 1)$ is associated with the common value signals. This weight is called the uncertainty parameter and is denoted $\gamma_{j}$ for the $j$ th auction. Intuitively, it denotes the degree of uncertainty in the common value of an object. For $j=1, \ldots, m$, we apply the following mean-preserving transformation to bidder $i$ 's (for $i=1, \ldots, n$ ) common value signal:

$$
v_{i j} \rightarrow \gamma_{j} v_{i j}+\left(1-\gamma_{j}\right) E\left(V_{j}\right)
$$

We apply this transformation because it leaves the expectation of the common value unchanged but increases its variance (i.e., its uncertainty). Note that $E\left(V_{j}\right)$ depends on the function $Q_{j}$, but not on the actual signals received by the bidders. Hence, the term $\left(1-\gamma_{j}\right) E\left(V_{j}\right)$ in Equation 19 is constant (i.e., it is independent of the bidders' signals) and is the same for all the bidders. In order to parameterize the uncertainty about the common value, the surplus is now defined as:

$$
S_{i j}\left(\gamma_{j}\right)=\gamma_{j} v_{i j} /(n-j+1)-c_{i j}
$$

Thus, if $\gamma_{j}$ is large, the common value component is large in comparison with the cost $\left(c_{i j}\right)$ and uncertainty is high. On the other hand, if $\gamma_{j}$ is low, the common value component is low in comparison with the cost $\left(c_{i j}\right)$ and the uncertainty is also low. Note that we do not consider the additive constant of Equation 19 in the surplus. This is because the additive constant merely causes an equal shift in the equilibrium bids of all the bidders. Hence, the relative positions of the bidders for the equilibrium of Theorem 1 is the same as the equilibrium for the transformation given in Equation 19.

For the parameterised definition of Equation 19, the work in [5] has studied the effect of $\gamma$ on the allocative efficiency for a single object. Below, we extend these results to our $m$ objects case.

The following theorems study the effect of uncertainty on the auction outcomes. For $j=1, \ldots, m$, we will denote the highest order statistic of the surplus for the $j$ th auction as $f_{j}^{n-j+1}\left(\gamma_{j}\right)$ and the second highest order statistic as $s_{j}^{n-j+1}\left(\gamma_{j}\right)$.
THEOREM 3. For $j=1, \ldots, m$, the expected cost of the winner for the jth auction is increasing in $\gamma_{j}$.

Proof. Consider the $j$ th (for $j=1, \ldots, m$ ) auction. For this auction, we know from Section 3, that the bidder with the highest surplus wins and pays $E\left(P_{w j}\right)$. Hence, the expected cost of the winner for this auction is:

$$
E\left(c_{w j}\right)=E\left(c \mid S\left(\gamma_{j}\right)=f_{j}^{n-j+1}\left(\gamma_{j}\right)\right)
$$

For a single object auction with $n$ bidders and uncertainty parameter $\gamma$, it has been shown that the expected cost of the winner $\left(E\left(c_{w}\right)=E\left(c \mid S(\gamma)=f^{n}(\gamma)\right)\right)$ is increasing in $\gamma$ [5]. The jth auction of our $m$ objects case is similar to the single object auction of [5], since the bidder with the highest surplus wins. The result of [5] therefore directly applies to the jth auction, and we get:

$$
E\left(c_{w j}\right)=E\left(c \mid S\left(\gamma_{j}\right)=f_{j}^{n-j+1}\left(\gamma_{j}\right)\right)
$$

is increasing in $\gamma_{j}$.

THEOREM 4. For $j=1, \ldots, m$, the probability $\left(P_{j}\left(\gamma_{j}\right)\right)$ that the bidder with the lowest cost wins the jth auction is decreasing in $\gamma_{j}$, with

$$
P_{j}(0)=1
$$

and

$$
\lim _{\gamma_{j} \rightarrow \infty} P_{j}\left(\gamma_{j}\right)=1 / n
$$

PROOF. To begin, consider the first auction. We know from Section 3, that the bidder with the highest surplus wins the first auction. The probability $\left(P_{1}\left(\gamma_{1}\right)\right)$ that this winner also has the lowest cost is:

$$
\begin{aligned}
P_{1}\left(\gamma_{1}\right)= & \operatorname{Prob}\left(S_{11}\left(\gamma_{1}\right)=\max _{i=1, \ldots, n} S_{i 1}\left(\gamma_{1}\right)\right. \\
& \left.\mid c_{11}=\min _{i=1, \ldots, n} c_{i 1}\right)
\end{aligned}
$$

If there is no uncertainty about the common value for the first auction (i.e., $\gamma_{1}=0$ ), the auction reduces to a private value auction. Hence, if $\gamma_{1}=0$, then $S_{i 1}\left(\gamma_{1}\right)=-c_{i 1}$ for $i=1, \ldots, n$. In other words, the surplus is negative for all the bidders. Hence, the maximum surplus is the one with lowest cost. Therefore, we get $P_{1}(0)=1$.

Equation 21 for $P_{1}\left(\gamma_{1}\right)$ can also be written as:

$$
\begin{aligned}
P_{1}\left(\gamma_{1}\right)= & \operatorname{Prob}\left(\gamma_{1} v_{21} / n-c_{21}<\gamma_{1} v_{11} / n-c_{11},\right. \\
& \gamma_{1} v_{31} / n-c_{31}<\gamma_{1} v_{11} / n-c_{11}, \ldots, \\
& \gamma_{1} v_{n 1} / n-c_{n 1}<\gamma_{1} v_{11} / n-c_{11} \\
& \left.\mid c_{11}=\min _{i=1, \ldots, n} c_{i 1}\right) \\
= & \operatorname{Prob}\left(c_{11}-c_{21}<\gamma_{1}\left(v_{11}-v_{21}\right) / n, \ldots,\right. \\
& c_{11}-c_{n 1}<\gamma_{1}\left(v_{11}-v_{n 1}\right) / n \\
& \left.\mid c_{11}-c_{21}<0, \ldots, c_{11}-c_{n 1}<0\right)
\end{aligned}
$$

Equation 22 shows that $P_{1}\left(\gamma_{1}\right)$ is decreasing in $\gamma_{1}$. As $\gamma_{1}$ tends to infinity, the condition

$$
c_{11}-c_{i 1}<\gamma_{1}\left(v_{11}-v_{i 1}\right) / n
$$

is true for $i=2, \ldots, n$. Hence, $P_{1}\left(\gamma_{1}\right)$ is the probability that $c_{11}-c_{21}<0, \ldots, c_{11}-c_{n 1}<0$, or the probability that $c_{11}<$ $c_{21}, \ldots, c_{11}<c_{n 1}$. The total number of possible orderings for the costs of $n$ bidders is $n$ !, out of which $c_{11}$ is the lowest in $(n-1)$ ! orderings. Hence, we have $\lim _{\gamma_{j} \rightarrow \infty} P_{j}\left(\gamma_{j}\right)=1 / n$ (which is the 
same as the probability that the lowest cost bidder wins if the object is allocated to a randomly selected bidder).

The above analysis also applies to the remaining $m-1$ auctions. This is because the winner for the $j$ th (for $j=1, \ldots, m$ ) auction is the bidder with the highest surplus for that auction. The only difference between the $m$ auctions is in the number of bidders $((n-$ $j+1)$ for the $j$ th auction).

THEOREM 5. For $j=1, \ldots, m$, the winner's expected profit for the $j$ th auction is non-decreasing in $\gamma_{j}$.

Proof. Consider the $j$ th (for $j=1, \ldots, m-1$ ) auction. For this auction, we vary $\gamma_{j}$ and study its effect on the winner's expected profit by keeping $\gamma_{j+1}, \ldots, \gamma_{m}$ constant. The winner's expected profit for this auction is:

$$
\begin{aligned}
E\left(\pi_{w j}\left(\gamma_{j}\right)\right)= & E\left(f_{j}^{n-j+1}\left(\gamma_{j}\right)\right)-E\left(s_{j}^{n-j+1}\left(\gamma_{j}\right)\right)+(23) \\
& \alpha_{j+1}
\end{aligned}
$$

(see Equation 13). This is of the same form as the winner's expected profit for the single object auction of [5]. Also, [5] shows that $E\left(f_{1}^{n}(\gamma)\right)-E\left(s_{1}^{n}(\gamma)\right)$ is non-decreasing in $\gamma$. This implies that $\alpha_{j+1}$ is nondecreasing in $\gamma$. It follows that for the first $m-1$ auctions of our $m$ objects case, the winner's expected profit is nondecreasing in $\gamma_{j}$. Since the last auction is similar to a single object auction, the above result applies to this auction also.

THEOREM 6. For $j=1, \ldots, m$, the total expected surplus (i.e., the expected efficiency) for the jth auction is decreasing in $\gamma_{j}$.

PROOF. Consider the $j$ th (for $j=1, \ldots, m$ ) auction (which has $n-j+1$ bidders). For this auction, we vary $\gamma_{j}$ and study its effect on the total expected surplus by keeping $\gamma_{k}$ (for $k=j+$ $1, \ldots, m)$ constant. The total expected surplus for this auction is:

$$
E\left(W_{j}\right)=E\left(V_{j}\right)-E\left(c_{w j}\right)
$$

(see Equation 15). We know from Theorem 3, that $E\left(c_{w j}\right)$ is increasing in $\gamma_{j}$. Furthermore, $E\left(V_{j}\right)$ does not change with $\gamma_{j}$, since the transformation in Equation 19 is mean-preserving. It follows that the total expected profit for the jth auction is decreasing in $\gamma_{j}$.

Theorem 7 follows from Theorems 5 and 6 and shows that, for each of the $m$ auctions, the auctioneer's expected revenue is decreasing in the uncertainty for that auction.

THEOREM 7. For $j=1, \ldots, m$, the expected revenue for the jth auction is decreasing in $\gamma_{j}$.

Proof. Consider the $j$ th (for $j=1, \ldots, m-1$ ) auction. Here, we vary $\gamma_{j}$ and study its effect on the expected revenue by keeping $\gamma_{k}$ (for $\left.k=j+1, \ldots, m\right)$ constant. The expected revenue for this auction is:

$$
\begin{aligned}
E\left(R_{j}\right)= & E\left(W_{j}\right)-\left(E\left(f_{j}^{n-j+1}\right)-E\left(s_{j}^{n-j+1}\right)+\right. \\
& \left.\alpha_{j+1}\right)
\end{aligned}
$$

(see Equation 16). We know from Theorem 6 that the total expected surplus for this auction (i.e., $E\left(W_{j}\right)$ ) is decreasing in $\gamma_{j}$. Also, we know from Theorem 5 that the winner's expected profit $\left(E\left(\pi_{w j}\left(\gamma_{j}\right)\right)=E\left(f_{j}^{n-j+1}\left(\gamma_{j}\right)\right)-E\left(s_{j}^{n-j+1}\left(\gamma_{j}\right)+\alpha_{j+1}\right)\right)$ is non-decreasing in $\gamma_{j}$. It follows that the expected revenue for the jth auction is decreasing in $\gamma_{j}$.

Since the auctioneer's expected revenue is decreasing in uncertainty, it is in the auctioneer's interest to reduce uncertainty. In the following section, we show that the auctioneer can do so by disclosing its information about the common values of the objects to all the bidders. We then show how competition affects efficiency.

\section{THE EFFECT OF DISCLOSURE OF THE AUCTIONEER'S INFORMATION}

The above analysis was conducted on the basis of the value and cost signals received by the $n$ bidders. However, since the objects for sale have a common value, the auctioneer too can have information about this value in the form of its own signal. If this is so, then for the single object case, it has been shown that the auctioneer can increase its revenue by making its information known to all the bidders ${ }^{3}$, relative to the situation where it does not give its information to them [5]. Given this, we extend this work by studying how the public disclosure of the auctioneer's information affects the expected revenue for $m$ sequential auctions.

For $j=1, \ldots, m$, let $v_{0 j}$ denote the auctioneer's signal for the common value $V_{j}$. For object $j=1, \ldots, m$ and bidder $i=$ $1 \ldots, n$, the common values (with the auctioneer's signal) are:

$$
V_{j}=\frac{1}{n-j+2} \sum_{i=0}^{n-j+2} v_{i j}
$$

In order to incorporate the auctioneer's extra piece of information, the surplus is now defined as:

$$
S_{i j}=v_{i j} /(n-j+2)-c_{i j}
$$

Theorem 8 shows that the auctioneer can increase its revenue by disclosing its common value signal to all the bidders.

THEOREM 8. Assume that the auctioneer discloses its information about the common value, $v_{0 j}($ for $j=1, \ldots, m)$, to all participating bidders just before the jth auction begins. Public disclosure of the auctioneer's information for the jth auction increases the expected revenue for that auction.

Proof. Consider the $j$ th (for $j=1, \ldots, m$ ) auction. Recall that the surplus with and without the auctioneer's signal is

$$
S_{i j}=v_{i j} /(n-j+2)-c_{i j}
$$

and

$$
S_{i j}=v_{i j} /(n-j+1)-c_{i j}
$$

respectively. In order to differentiate between these two surpluses, we use the parameterised definition of Equation 20 (i.e., $S_{i j}\left(\gamma_{j}\right)=$ $\left.\gamma_{j} v_{i j} /(n-j+1)-c_{i j}\right)$. Let

$$
\gamma_{a}=1 \text { and } \gamma_{b}=(n-j+1) /(n-j+2)
$$

For the jth auction, the surplus without the auctioneer's signal is $S_{i j}\left(\gamma_{a}\right)$, and with it is $S_{i j}\left(\gamma_{b}\right)$. From Theorem 7, we know that the expected revenue for the jth auction is decreasing in $\gamma_{j}$. It follows that the expected revenue for the jth auction is higher with the auctioneer's signal than without it (since $\gamma_{a}>\gamma_{b}$ ).

\section{THE EFFECT OF COMPETITION}

The difference between auctions in an all-human setting and those in an agent based setting is in terms of the competition (i.e., the number of bidders) they generate. Online auctions make participation for bidders easier, thereby increasing competition [14]. The following theorem shows how competition affects auction efficiency.

\footnotetext{
${ }^{3}$ This work shows that such a disclosure of information reduces uncertainty about the common value. This is because, if the uncertainty is high, the bidders bid less in order to avoid the problem of the winner's curse. But if the uncertainty is low, the bidders bid higher because they are now more certain about the common value. Our work extends these results to the $m$ objects case.
} 
THEOREM 9. An increase in competition (i.e., the number of bidders for the first auction) raises the efficiency of the $m$ auctions. Efficiency is maximized as the number of bidders goes to infinity.

PROOF. Consider two cases: one with $n$ bidders for the first auction and the other with $n+\delta$ bidders, where $\delta \in Z_{+}$. For the $j$ th (for $j=1, \ldots, m$ ) auction, the surplus for these two cases is

$$
S_{i j}=v_{i j} /(n-j+1)-c_{i j}
$$

and

$$
S_{i j}=v_{i j} /(n-j+1+\delta)-c_{i j}
$$

respectively. Again we use the parameterised definition $S_{i j}\left(\gamma_{j}\right)=$ $\gamma_{j} v_{i j} /(n-j+1)-c_{i j}$ for the surplus. Let

$$
\gamma_{a}=1 \text { and } \gamma_{b}=(n-j+1) /(n-j+1+\delta)
$$

From Theorem 7, we know that the expected revenue for the jth auction is decreasing in $\gamma_{j}$. Since $\gamma_{a}>\gamma_{b}$, the expected revenue is higher for the $(n+\delta)$ bidders case than that for $n$ bidders. Thus, the first part of the theorem follows.

As $n \rightarrow \infty$, surplus reduces to $-c_{i j}$ and the surplus is negative for all the bidders for all the objects. Hence, the maximum surplus is the one with the lowest cost. Recall that for each of the $m$ auctions, the bidder with the highest surplus wins. Since the bidder with the highest surplus also has the lowest cost, the efficiency is maximum.

Our analysis provides two key results. First, the efficiency of an auction can be increased if the auctioneer discloses its information about the common value for that auction (as per Theorem 8). Second, the efficiency of each auction in a sequence is higher in the context of software agents than the corresponding auction in a human setting. This is because the use of software agents leads to relatively more bidders participating in the auctions [14]. Since the number of bidders (i.e., $n$ in Equation 20) increases, the effect of the common value part decreases in comparison with the private value part, which is analogous to a reduction in uncertainty. This leads to higher efficiency (see Theorem 9).

\section{RELATED WORK}

Existing work on sequential auctions includes [12, 18, 11, 10, 2]. Our work differs from these in two ways. First, this existing work focuses on the study of the dynamics of the selling price in sequential auctions for identical objects. Second, it focuses on objects that are either exclusively private value or exclusively common value. For instance, Ortega-Reichert [12] determined the equilibrium for sequential auctions for two private value objects using the first price rules. Weber [18] showed that in sequential auctions of identical objects with risk neutral bidders who hold independent private values, the expected selling price is the same for each auction. On the other hand, Milgrom and Weber [11] studied sequential auctions in an interdependent values model with affiliated ${ }^{4}$ signals. They showed that expected selling prices have a tendency to drift upward. Mc Afee and Vincent [10] consider two identical private value objects and using the second price sealed bid rules, they show that prices increase in later auctions. Bernhardt and Scoones [2] consider two identical private value objects and show that the selling price for the second auction can be lower than the first. Our model is a generalization of [2] since we consider $n>2$ objects with

\footnotetext{
${ }^{4}$ Affiliation is a form of positive correlation. Let $X_{1}, X_{2}, \ldots, X_{n}$ be a set of positively correlated random variables. Positive correlation roughly means that if a subset of $X_{i} \mathrm{~s}$ are large, then this makes it more likely that the remaining $X_{j}$ s are also large.
}

both common and private values. Furthermore, while [2] focuses on the dynamics of the selling price, we concentrate on the efficiency property of auctions.

A key difference between the exclusive common/private value auctions and the those that have both elements is in terms of their efficiency. For the former category, a number of auction mechanisms have been developed that have the property of efficiency. For example, in the private values context, Vickrey [17] showed that the equilibrium bids are increasing in the bidders' values, and hence, the object is awarded to the bidder that values it the most (i.e., the auction is efficient). And for common value auctions, all bidders value the object the same, so any allocation rule is trivially efficient. However, for auctions with multi-dimensional signals, Maskin [9] showed that it is impossible to allocate an object efficiently. Dasgupta and Maskin [3] extended Maskin's earlier work and showed that the Vickrey auction is inefficient for multidimensional signals. Also, in the context of two dimensional signals Pesendorfer [13] showed that efficiency varies with the number of bidders. Since two signals are necessary to model objects with private and common value elements, it follows that auctions for such objects are, in general, inefficient.

Given this, it is important to investigate the property of efficiency since our model involves two-dimensional signals (one for value and the other for cost). To this end, Theorem 6 shows that the expected efficiency (measured in terms of the total expected surplus) for each of the $m$ auctions decreases with the uncertainty for that auction. Also, Theorem 4 shows that, in the limit (i.e., as $\gamma_{j} \rightarrow \infty$ ), the uncertainty about the common value is so large as to override the private value component (see Equation 20), an auction is no more efficient than a random allocation rule. At the other extreme, when there is no uncertainty about the common value (i.e., $\gamma_{j}=0$ ), an auction reduces to an efficient private value auction. For our model, uncertainty and competition are related. As competition (i.e., $n$ in Equation 20) increases, the effect of the common value part decreases in comparison with the private value part, which is analogous to a reduction in uncertainty. This leads to higher efficiency (see Theorem 9).

\section{CONCLUSIONS AND FUTURE WORK}

This paper has analyzed sequential auctions for objects with private and common values, in an uncertain information setting. We first determined equilibrium strategies for each auction in a sequence. The lack of complete information (i.e., the uncertainty about the common values) leads to inefficiencies in the auctions. On the basis of the equilibrium, we determined the relation between uncertainty and efficiency. We showed that, for each auction in a sequence, efficiency decreases with uncertainty. In the limit, when uncertainty is very high, the auction mechanism is no better than a random allocation of the object to a bidder. At the other extreme, when there is no uncertainty, the auctions reduce to efficient private value auctions. We also showed that efficiency is improved if the auctioneer makes its information about the common values known to all the bidders, and also if competition increases.

Our present focus was on the efficiency of sequential auctions. But apart from efficiency, there are other important issues that need to be addressed. First, is the question of the order in which the auctions should be conducted (where this is a choice). For such cases, we would like to determine the order that maximizes total expected revenue across all the auctions in a sequence. Second, in this work, we used the English auction form which is by far the most common form in practice. However, in order to generalize our results, we intend to extended the analysis to other auction forms. Finally, we will extend our analysis to the case where each bidder 


\section{REFERENCES}

[1] J. P. Benoit and V. Krishna. Multiple-object auctions with budget constrained bidders. Review of Economic Studies, 68:155-179, 2001.

[2] D. Bernhardt and D. Scoones. A note on sequential auctions. American Economic Review, 84(3):653-657, 1994.

[3] P. Dasgupta and E. Maskin. Efficient auctions. Quarterly Journal of Economics, 115:341-388, 2000.

[4] W. Elmaghraby. The importance of ordering in sequential auctions. Management Science, 49(5):673-682, 2003.

[5] J. K. Goeree and T. Offerman. Competitive bidding in auctions with private and common values. The Economic Journal, 113(489):598-613, 2003.

[6] B. Katzman. A two stage sequential auction with multi-unit demands. Journal of Economic Theory, 86:77-99, 1999.

[7] V. Krishna. Auction Theory. Academic Press, 2002.

[8] J. Laffont. Game theory and empirical economics: The case of auction data. European Economic Review, 41:1-35, 1997.

[9] E. Maskin. Auctions and privatization. In Privatization, pages 115-136. Kiel: Institut fur Weltwirtschaften der Universitat Kiel, 1992.

[10] R. P. McAfee and D. Vincent. The declining price anomaly. Journal of Economic Theory, 60:191-212, 1993.

[11] P. Milgrom and R. J. Weber. A theory of auctions and competitive bidding II. In The Economic Theory of Auctions. Edward Elgar, Cheltenham, U.K, 2000.

[12] A. Ortega-Reichert. Models of competitive bidding under uncertainty. Technical Report 8, Stanford University, 1968.

[13] W. Pesendorfer and J. M. Swinkels. Efficiency and information aggregation in auctions. American Economic Review, 90:499-525, 2000.

[14] E. J. Pinker, A. Seidman, and Y. Vakrat. Managing online auctions: Current business and research issues. Management Science, 49(11):1457-1484, 2003.

[15] C. Pitchik and A. Schotter. Perfect equilibria in budget-constrained sequential auctions: An experimental study. Rand Journal of Economics, 19:363-388, 1988.

[16] T. Sandholm and S. Suri. BOB: Improved winner determination in combinatorial auctions and generalizations. Artificial Intelligence, 145:33-58, 2003.

[17] W. Vickrey. Counterspeculation, auctions and competitive sealed tenders. Journal of Finance, 16:8-37, 1961.

[18] R. J. Weber. Multiple-object auctions. In R. Engelbrecht-Wiggans, M. Shibik, and R. M. Stark, editors, Auctions, bidding, and contracting: Uses and theory, pages 165-191. New York University Press, 1983.

[19] M. P. Wellman, W. E. Walsh, P. R. Wurman, and J. K. McKie-Mason. Auction protocols for decentralised scheduling. Games and Economic Behavior, 35:271-303, 2001.

[20] R. E. Wiggans and C. M. Kahn. Multi-unit pay your-bid auctions with variable rewards. Games and Economic Behavior, 23:25-42, 1998. 